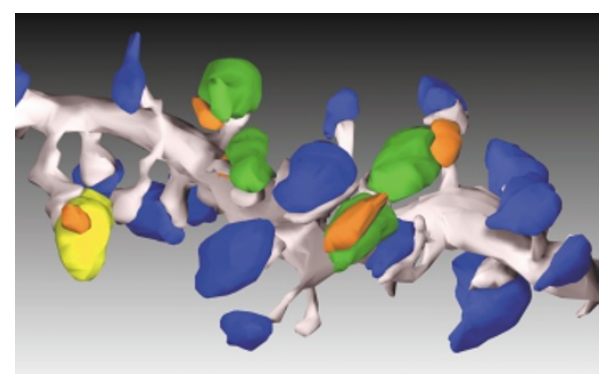

3D reconstruction of a dendrite from a hippocampal neuron (white) showing synaptic connections (SSBs, blue; different-cell MSBs, green; same-cell MSBs, yellow; spines from other neurons; orange). Photograph courtesy of Catherine Woolley, Northwestern University, Evanston, Illinois, USA. ㅇ (2001) National Academy of Sciences USA.

SYNAPTIC PLASTICITY

\section{Strength in numbers}

Although plasticity in the adult brain is thought to be largely achieved by modifying the strength of existing synapses, there is increasing evidence that plastic changes might also be induced by the formation of new synaptic contacts. For example, it has been shown that in the female rat hippocampus, new synaptic connections are built up and broken down in a highly dynamic fashion during the 4-day oestrous cycle. Hormones play an important part in regulating this process, and oestrogen in particular has been shown to induce synaptogenesis. Do the newly generated synapses serve simply to increase the number of contacts between cells that were already connected? Not according to a new report by Yankova et al., which presents compelling evidence that oestrogen can induce the formation of synapses between previously unconnected cells.

The authors analysed individual synaptic boutons in the hippocampus and assessed whether they synapsed onto a single dendritic spine (single synapse boutons or SSBs), or two or more spines (multiple synapse boutons or MSBs). Using ovariectomized rats, they compared the density of SSBs and MSBs on hippocampal dendrites in the presence and absence of oestrogen, and asked whether the MSBs contacted multiple spines on a single cell or on different cells. To this end, they labelled individual layer CAl pyramidal cells with biocytin, which fills the entire cell, including all of the dendritic spines. A bouton was classed as a different-cell MSB if it synapsed onto a labelled spine plus at least one unlabelled spine. They reconstructed individual labelled dendrites from a series of ultrathin sections and measured the distribution of different classes of synaptic contact. They showed that oestrogen treatment doubled the number of different-cell MSBs but had no significant effect on SSBs or same-cell MSBs. So, the predominant effect of oestrogen was the generation of synapses between previously unconnected neurons, thereby increasing the diversity of synaptic connections.

What is the purpose of the increased plasticity in response to oestrogen? We can only speculate, but these new findings nevertheless have intriguing implications. Oestrogen has been shown to improve cognitive function in humans, and it might even help to delay the progression of Alzheimer's disease. However, it also reduces the threshold for epileptic seizures in the hippocampus. This new study provides possible mechanisms to explain both the beneficial and deleterious effects; the ability to generate new synaptic contacts in the hippocampus might be an asset for learning and memory, but increased connective diversity might also help to propagate seizures by raising the level of synchronous neuronal firing. The findings of Yankova et al. not only add a new dimension to our concepts of hippocampal plasticity, but might also aid in the development of new strategies to treat neurological disorders.

\section{(2) References and links} ORIGINAL RESEARCH PAPER Yankova, M. etal. Estrogen increases synaptic connectivity between single presynaptic inputs and multiple postsynaptic CA1 pyramidal cells: a serial electron-microscopic study. Proc. Natt Acad. Sci. USA 98, 3525-3530 (2001)

FURTHER READING Wooley, C. S. Effects of estrogen in the CNS. Curr. Opin. Neurobiol. 9 , 349-354 (1999) | Breedlove, S. M. \& Jordan, C. L. The increasingly plastic, hormone-responsive adult brain. Proc. Natl Acad. Sci. USA 98, 2956-2957 (2001)

ENCYCLOPEDIA OF LIFE SCIENCES

Oestrogens, mood and cognition

H I G H L I G H T S

\title{
LANGUAGE
}

\section{Mamma mia!}

Even if you've never learned Italian, the pronunciation of the exclamation above can seem more instinctive than some of the quirkier words in the English language, such as 'sobriquet'. This is a characteristic of written languages as a whole: some, such as Italian, have a 'shallow' orthography - letters in the alphabet alone or in combination usually map uniquely to speech sounds. Others such as English are much more ambiguous (have a 'deep' orthography); for example, the 'o' in tomb, bomb or comb is pronounced differently in each word. These differences in the ease of reading for different languages have important implications for the study of dyslexia, as the variable nature and prevalence of this reading disorder across languages have led to doubts that it stems from a particular cognitive deficit. But now, by contrasting dyslexic and normal readers in languages with deep (English and French) and shallow (Italian) orthographies, a multinational group of researchers have provided strong evidence that dyslexia has a unitary neurocognitive basis.

The authors studied the differences in performance of reading and phonological tasks in English, French and Italian people with dyslexia and in normal controls matched for age and IQ. Tests were in the native language and included assessments of reaction time to words and non-words, digit-naming skill and auditory short-term memory for short and long words. The Italian dyslexic people performed consistently better when reading than their English and French counterparts, as might be expected given the shallower orthography of Italian. But crucially, despite the differences in performance across languages, the impairment in the performance of the dyslexic people relative to their controls was independent of language. Furthermore, positron-emission tomography studies of dyslexics and controls from the three countries revealed that reduced activation in the left-middle, inferior and superior temporal cortex and in the middle occipital gyrus was a feature common to dyslexia for word reading in all three language groups. So, as the authors conclude, it seems that "dyslexia has a universal basis in the brain and can be characterized by the same neurocognitive deficit", with the effect of this deficit being less in languages with shallow orthography.

Peter Kirkpatrick

(1) References and links

ORIGINAL RESEARCH PAPER Paulesu, E. et al. Dyslexia: cultural diversity and biological unity. Science 291, 2165-2167 (2001)

FURTHER READING Paulesu, E. et al. A cultural effect on brain function. Nature Neurosci. 3, 91-96 (2000) 